\title{
Tightly coupled position and attitude determination with two low-cost GNSS receivers
}

\author{
Patrick Henkel $(*, * *)$ and Michele Iafrancesco $(*, * *)$ \\ * Technische Universität München, Munich, Germany \\ ** Advanced Navigation Solutions - ANavS, Munich, Germany \\ patrick.henkel@tum.de
}

\begin{abstract}
A precise position and attitude information is essential for autonomous driving of any vehicle. Low-cost GNSS receivers and antennas can provide a precise attitude and drift-free position information. However, severe code multipath, frequent half cycle slips and losses of lock might temporarily reduce the accuracy. Inertial sensors are robust to GNSS signal interruption and very precise over short time frames, which enables a reliable cycle slip correction. However, low-cost inertial sensors suffer from a substantial drift. In this paper, we propose a tightly coupled position and attitude determination method for two low-cost GNSS receivers, a gyroscope and an accelerometer. It improves classical tightly coupled solutions by including a synchronization correction, by the estimation of the code multipath for each satellite and receiver, and by the additional determination of satellite-satellite single difference ambiguities. The proposed method was verified in a test drive. We obtained a heading with an accuracy of $0.25^{\circ} /$ baseline length [m] and an absolute position with an accuracy of $1 \mathrm{~m}$.
\end{abstract}

Index Terms-Navigation, Attitude determination, Tight coupling, Kalman Filtering.

\section{INTRODUCTION}

The sensor fusion of GNSS and INS measurements is very attractive as both sensors complement each other. The carrier phase, pseudorange, Doppler, angular rate and acceleration measurements can be combined to estimate the absolute 3D position, the absolute $3 \mathrm{D}$ velocity, the absolute $3 \mathrm{D}$ acceleration, the heading and rate of heading, the pitch and rate of pitch, the sat.-sat. single difference ambiguities, the sat.-sat. double difference (DD) integer ambiguities, the sat.-sat. single difference code multipath for each receiver and satellite, the biases of the gyroscope and the biases of the accelerometer. A Kalman filter is typically used as it additionally considers the receiver dynamics and as it enables a real-time implementation. Tropospheric and ionospheric delays are corrected with correction data and/ or a model to improve the absolute position accuracy. A tight coupling with integer ambiguity fixing has not yet been performed with low-cost GNSS receivers in kinematic environments. The DD ambiguities are no longer integer valued due to the lack of a precise synchronization of GNSS receivers. Additionally, the code multipath of lowcost GNSS receivers is significantly larger than for geodetic receivers.

In this paper, we first describe an improved model for the satellite-satellite single difference phase, code and Doppler measurements of low-cost GNSS receivers. A synchronization correction is derived to improve the positioning accuracy and to restore the integer property of DD ambiguities. Subsequently, we describe the sensor fusion with an extended Kalman filter [1]. Finally, the results of a test drive with two u-blox LEA 6T GPS receivers, a 3D accelerometer and 3D gyroscope are provided.

\section{Measurement Models}

In this section, we describe our models for the carrier phase, pseudorange and Doppler measurements of low-cost GNSS receivers as well as our models for the measurements of a low-cost gyroscope and accelerometer.

\section{A. Models for GNSS measurements}

Low cost GNSS receivers do not provide the option of external synchronization. Their oscillators drift and this is only compensated at millisecond-level. As satellites move with a speed of $3 \mathrm{~km} / \mathrm{s}$, the satellite movement within the time of the receiver clock offset can not be neglected. Therefore, we model the GNSS measurements at the time of signal reception, i.e. the clock offset $\delta t_{r}$ of the $r$-th receiver is explicitly considered in the satellite position models. The single difference (SD) carrier phase measurement of receiver $r \in\{1,2\}$ between satellites $k$ and $l$ is modeled as

$$
\begin{aligned}
& \lambda \tilde{\varphi}_{r}^{k, l}\left(t_{n}+\delta t_{r}\left(t_{n}\right)\right) \\
& =\vec{e}_{r}^{k}\left(t_{n}+\delta t_{r}\left(t_{n}\right)\right)\left(\vec{x}_{r}\left(t_{n}+\delta t_{r}\left(t_{n}\right)\right)-\vec{x}^{k}\left(t_{n}+\delta t_{r}\left(t_{n}\right)\right)\right) \\
& \quad-\vec{e}_{r}^{l}\left(t_{n}+\delta t_{r}\left(t_{n}\right)\right)\left(\vec{x}_{r}\left(t_{n}+\delta t_{r}\left(t_{n}\right)\right)-\vec{x}^{l}\left(t_{n}+\delta t_{r}\left(t_{n}\right)\right)\right) \\
& \quad+c \delta t_{r}^{k, l}\left(t_{n}\right)-I_{r}^{k, l}\left(t_{n}\right)+T_{r}^{k, l}\left(t_{n}\right) \\
& \quad+\lambda N_{r}^{k, l}+\lambda / 2 \Delta N_{r}^{k, l}+\lambda \beta^{k, l}+\varepsilon_{r}^{k, l}\left(t_{n}\right),
\end{aligned}
$$

with the line of sight vector $\vec{e}_{r}^{k}$ pointing from satellite $k$ to receiver $r$, the receiver position $\vec{x}_{r}$, the satellite position $\vec{x}^{k}$, the speed of light $c$, the satellite clock offset $\delta t_{r}^{k}$, the wavelength $\lambda$, the slant ionospheric delay $I_{r}^{k}$, the slant tropospheric delay $T_{r}^{k}$, the integer ambiguity $N_{r}^{k}$, the half cycle slip $\Delta N_{r}^{k}$, the satellite phase bias $\beta^{k}$ and the phase noise $\varepsilon_{r}^{k}$. We use a similar model for the SD code measurements, i.e.

$$
\begin{aligned}
& \tilde{\rho}_{r}^{k, l}\left(t_{n}+\delta t_{r}\left(t_{n}\right)\right) \\
& =\vec{e}_{r}^{k}\left(t_{n}+\delta t_{r}\left(t_{n}\right)\right)\left(\vec{x}_{r}\left(t_{n}+\delta t_{r}\left(t_{n}\right)\right)-\vec{x}^{k}\left(t_{n}+\delta t_{r}\left(t_{n}\right)\right)\right) \\
& \quad-\vec{e}_{r}^{l}\left(t_{n}+\delta t_{r}\left(t_{n}\right)\right)\left(\vec{x}_{r}\left(t_{n}+\delta t_{r}\left(t_{n}\right)\right)-\vec{x}^{l}\left(t_{n}+\delta t_{r}\left(t_{n}\right)\right)\right) \\
& \quad+c \delta t_{r}^{k, l}\left(t_{n}\right)+I_{r}^{k, l}\left(t_{n}\right)+T_{r}^{k, l}\left(t_{n}\right)+b^{k, l} \\
& \quad+\Delta \rho_{\mathrm{MP}_{r}^{k, l}}\left(t_{n}\right)+\eta_{r}^{k, l}\left(t_{n}\right)
\end{aligned}
$$


with the satellite code bias $b^{k}$, the code multipath $\Delta \rho_{\mathrm{MP}_{r}^{k}}$ and the code noise $\eta_{r}^{k}$. The satellite positions and clock offsets and atmospheric errors are known and can be brought to the left side. As this rearrangement can be made for both phase and code measurements, we limit ourselves to the phase measurements. However, we distinguish between both GNSS receivers. For the first receiver, the rearranged SD phase measurements are given by

$$
\begin{aligned}
& \lambda \varphi_{1}^{k, l}\left(t_{n}+\delta t_{1}\left(t_{n}\right)\right) \\
&:= \lambda \tilde{\varphi}_{1}^{k, l}\left(t_{n}+\delta t_{1}\left(t_{n}\right)\right)+\vec{e}_{1}^{k}\left(t_{n}+\delta t_{1}\left(t_{n}\right)\right) \vec{x}^{k}\left(t_{n}+\delta t_{1}\left(t_{n}\right)\right) \\
&-\vec{e}_{1}^{l}\left(t_{n}+\delta t_{1}\left(t_{n}\right)\right) \vec{x}^{l}\left(t_{n}+\delta t_{1}\left(t_{n}\right)\right)-c \delta t_{1}^{k, l}\left(t_{n}\right) \\
&+I_{1}^{k, l}\left(t_{n}\right)-T_{1}^{k, l}\left(t_{n}\right) \\
&= \vec{e}_{1}^{k, l}\left(t_{n}+\delta t_{1}\left(t_{n}\right)\right)\left(\vec{x}_{1}\left(t_{n}+\delta t_{1}\left(t_{n}\right)\right)\right)+\lambda\left(N_{1}^{k, l}+\beta^{k, l}\right) \\
&+\lambda / 2 \Delta N_{1}^{k, l}+\varepsilon_{1}^{k, l}\left(t_{n}\right) .
\end{aligned}
$$

For the second receiver, we express the position $\vec{x}_{2}$ in terms of the position $\vec{x}_{1}$ of the first receiver and the baseline vector given by

$$
\vec{b}_{12}=\vec{x}_{1}-\vec{x}_{2}=l \cdot\left(\begin{array}{c}
\sin (\psi) \cos (\theta) \\
\cos (\psi) \cos (\theta) \\
\sin (\theta)
\end{array}\right),
$$

with the baseline length $l$, the heading $\psi$ and the pitch angle $\theta$. An initial heading information might be obtained from magnetometers, for further details we refer to [2]. The SD ambiguities $N_{2}^{k, l}$ are also written in terms of $N_{1}^{k, l}$ and the DD ambiguities $N_{1,2}^{k, l}=N_{1}^{k, l}-N_{2}^{k, l}$ to benefit from the integer property of the latter ones. The receiver and satellite positions are considered at time $t_{n}+\delta t_{1}\left(t_{n}\right)$ and the movements of the receiver and satellites within $\delta t_{1}\left(t_{n}\right)-\delta t_{2}\left(t_{n}\right)$ are considered in the synchronization corrections $c_{1,2}^{k, l}\left(t_{n}\right)$, i.e.

$$
\begin{aligned}
& \lambda \varphi_{2}^{k, l}\left(t_{n}+\delta t_{2}\left(t_{n}\right)\right):=\lambda \tilde{\varphi}_{2}^{k, l}\left(t_{n}+\delta t_{2}\left(t_{n}\right)\right) \\
& +\vec{e}_{1}^{k}\left(t_{n}+\delta t_{1}\left(t_{n}\right)\right) \vec{x}^{k}\left(t_{n}+\delta t_{1}\left(t_{n}\right)\right) \\
& -\vec{e}_{1}^{l}\left(t_{n}+\delta t_{1}\left(t_{n}\right)\right) \vec{x}^{l}\left(t_{n}+\delta t_{1}\left(t_{n}\right)\right)-c \delta t_{2}^{k, l}\left(t_{n}\right) \\
& +I_{2}^{k, l}\left(t_{n}\right)-T_{2}^{k, l}\left(t_{n}\right) \\
& =\vec{e}_{1}^{k, l}\left(t_{n}+\delta t_{1}\left(t_{n}\right)\right)\left(\vec{x}_{1}\left(t_{n}+\delta t_{1}\left(t_{n}\right)\right)-\vec{b}_{12}\left(t_{n}+\delta t_{1}\left(t_{n}\right)\right)\right) \\
& \quad+\lambda\left(N_{1}^{k, l}-N_{1,2}^{k, l}+\beta^{k, l}\right)+\lambda / 2 \Delta N_{2}^{k, l} \\
& \quad+c_{1,2}^{k, l}\left(t_{n}\right)+\varepsilon_{2}^{k, l}\left(t_{n}\right),
\end{aligned}
$$

with the synchronization correction

$$
\begin{aligned}
& c_{1,2}^{k, l}\left(t_{n}\right)= \\
& \vec{e}_{2}^{k}\left(t_{n}+\delta t_{2}\left(t_{n}\right)\right)\left(\vec{x}_{2}\left(t_{n}+\delta t_{2}\left(t_{n}\right)\right)-\vec{x}^{k}\left(t_{n}+\delta t_{2}\left(t_{n}\right)\right)\right) \\
& \quad-\vec{e}_{2}^{l}\left(t_{n}+\delta t_{2}\left(t_{n}\right)\right)\left(\vec{x}_{2}\left(t_{n}+\delta t_{2}\left(t_{n}\right)\right)-\vec{x}^{l}\left(t_{n}+\delta t_{2}\left(t_{n}\right)\right)\right) \\
& -\vec{e}_{1}^{k}\left(t_{n}+\delta t_{1}\left(t_{n}\right)\right)\left(\vec{x}_{2}\left(t_{n}+\delta t_{1}\left(t_{n}\right)\right)-\vec{x}^{k}\left(t_{n}+\delta t_{1}\left(t_{n}\right)\right)\right) \\
& +\vec{e}_{1}^{l}\left(t_{n}+\delta t_{1}\left(t_{n}\right)\right)\left(\vec{x}_{2}\left(t_{n}+\delta t_{1}\left(t_{n}\right)\right)-\vec{x}^{l}\left(t_{n}+\delta t_{1}\left(t_{n}\right)\right)\right) .
\end{aligned}
$$

This synchronization correction can be determined from rough code-only least-squares estimates of the receiver position and receiver clock offsets. The GNSS receivers also track the Doppler frequencies, which are modeled as

$$
\begin{aligned}
\tilde{f}_{\mathrm{D}_{r}}^{k, l}= & -f_{\mathrm{c}}\left(\left(\vec{e}_{r}^{k}\right)^{\mathrm{T}}\left(\vec{v}_{r}-\vec{v}^{k}\right) / c-\left(\vec{e}_{r}^{l}\right)^{\mathrm{T}}\left(\vec{v}_{r}-\vec{v}^{l}\right) / c\right) \\
& +f_{\mathrm{c}} \delta \dot{\tau}^{k, l}+\varepsilon_{f_{\mathrm{D} r}^{k, l}}
\end{aligned}
$$

with the carrier frequency $f_{\mathrm{c}}$, the receiver velocity $\vec{v}_{r}$, the satellite velocity $\vec{v}^{k}$, the clock drift rate $\delta \dot{\tau}^{k}$ and the frequency noise $\varepsilon_{f_{\mathrm{D}_{r}}^{k, l}}$. The known terms are again brought to the left side:

$$
\begin{aligned}
f_{\mathrm{D}_{r}}^{k, l}: & =\tilde{f}_{\mathrm{D}_{r}}^{k, l}-f_{\mathrm{c}}\left(\vec{e}_{r}^{k}\right)^{\mathrm{T}} \vec{v}^{k} / c+f_{\mathrm{c}}\left(\vec{e}_{r}^{l}\right)^{\mathrm{T}} \vec{v}^{l} / c-f_{\mathrm{c}} \delta \dot{\tau}^{k, l} \\
& =-f_{\mathrm{c}}\left(\vec{e}_{r}^{k, l}\right)^{\mathrm{T}} \vec{v}_{r} / c+\varepsilon_{f_{\mathrm{D}_{r}}^{k, l}} .
\end{aligned}
$$

The velocity $\vec{v}_{2}$ of the second receiver can also be expressed in terms of the velocity of the first receiver and the attitude angles, i.e.

$$
\begin{aligned}
\vec{v}_{2}= & \dot{\vec{x}}_{2}=\vec{v}_{1}-\dot{\vec{b}}_{12} \\
= & \dot{\vec{x}}_{1}-l \cdot\left(\left(\begin{array}{c}
\cos (\psi) \cos (\theta) \\
-\sin (\psi) \cos (\theta) \\
0
\end{array}\right) \dot{\psi}\right. \\
& \left.+\left(\begin{array}{c}
-\sin (\psi) \sin (\theta) \\
-\cos (\psi) \sin (\theta) \\
\cos (\theta)
\end{array}\right) \dot{\theta}\right)
\end{aligned}
$$

with $\dot{\psi}$ and $\dot{\theta}$ being the rate of heading and rate of pitch.

\section{B. Models for inertial measurements}

Inertial sensors provide high-rate acceleration and angular rate measurements, which are not affected by GNSS signal reception conditions and enable a reliable detection and correction of cycle slips for kinematic receivers. The acceleration and angular rate are sensed in the sensor-fixed (s-) frame, which is centered at the sensor's chip and aligned with the principal axes of the chip. We assume that the s-frame is aligned with the body-fixed (b-) frame, which is centered at the vehicle and aligned with the longitudinal and transversal axes of the vehicle.

As GNSS and inertial measurements are obtained in different frames, a frame transformation is needed. We use the e-frame (also ECEF frame) for the sensor fusion. It is centered at the Earth's centre with the $\mathrm{X}$-axis pointing in the equatorial plane towards the $0^{\circ}$ meridian and the $\mathrm{z}$-axis pointing towards the geographic north pole. The navigation (n-) frame is centered at the vehicle and aligned with the East, North and Up direction. The $\mathrm{n}$-frame serves as a reference frame for the attitude of the vehicle. The acceleration measurement is provided in the $b$-frame and modeled as

$$
\begin{aligned}
a^{(\mathrm{b})}\left(t_{n}\right)= & R_{\mathrm{n}}^{\mathrm{b}}\left(t_{n}\right) R_{\mathrm{e}}^{\mathrm{n}}\left(t_{n}\right) a^{(\mathrm{e})}\left(t_{n}\right)+b_{a}^{(\mathrm{b})}\left(t_{n}\right) \\
& +g\left(\begin{array}{c}
\sin \left(\theta\left(t_{n}\right)\right) \\
\sin \left(\varphi\left(t_{n}\right)\right) \\
\cos \left(\theta\left(t_{n}\right)\right) \cos \left(\varphi\left(t_{n}\right)\right)
\end{array}\right)+\varepsilon_{a}^{(\mathrm{b})}\left(t_{n}\right),
\end{aligned}
$$

with the rotation matrices $R_{\mathrm{e}}^{\mathrm{n}}$ and $R_{\mathrm{n}}^{\mathrm{b}}$, the acceleration $a^{(\mathrm{e})}$ in the e-frame, the acceleration biases $b_{a}^{(\mathrm{b})}$ of the sensor in the 
b-frame, the gravitational acceleration $g$, the pitch angle $\theta$, the roll angle $\varphi$ and the measurement noise $\varepsilon_{a}^{(\mathrm{b})}$. The rotation from the e-frame into the n-frame depends on the latitude $\varphi_{1}$ and longitude $\lambda_{1}$ of receiver 1 and is given by

$$
R_{\mathrm{e}}^{\mathrm{n}}\left(t_{n}\right)=R_{1}\left(\pi / 2-\varphi_{1}\left(t_{n}\right)\right) R_{3}\left(\pi / 2+\lambda_{1}\left(t_{n}\right)\right) .
$$

The rotation from the $\mathrm{n}$-frame into the b-frame depends on the heading $\psi$ and pitch $\theta$ of the vehicle and is given by

$$
R_{\mathrm{n}}^{\mathrm{b}}\left(t_{n}\right)=R_{2}\left(-\theta\left(t_{n}\right)\right) R_{3}\left(\pi / 2-\psi\left(t_{n}\right)\right) .
$$

The angular rate measurements are also biased and modeled in the b-frame as

$$
\begin{aligned}
& {\left[\dot{\psi}^{(\mathrm{b})}\left(t_{n}\right), \dot{\theta}^{(\mathrm{b})}\left(t_{n}\right), \dot{\varphi}^{(\mathrm{b})}\left(t_{n}\right)\right]^{\mathrm{T}}} \\
& =\left[\dot{\psi}\left(t_{n}\right), \dot{\theta}\left(t_{n}\right), \dot{\varphi}\left(t_{n}\right)\right]^{\mathrm{T}}+\left[b_{\dot{\psi}}^{(\mathrm{b})}\left(t_{n}\right), b_{\dot{\theta}}^{(\mathrm{b})}\left(t_{n}\right), b_{\dot{\varphi}}^{(\mathrm{b})}\left(t_{n}\right)\right]^{\mathrm{T}} \\
& \quad+\left[\varepsilon_{\dot{\psi}}\left(t_{n}\right), \varepsilon_{\dot{\theta}}\left(t_{n}\right), \varepsilon_{\dot{\varphi}}\left(t_{n}\right)\right]^{\mathrm{T}}
\end{aligned}
$$

with the rate of heading $\dot{\psi}$, the rate of pitch $\dot{\theta}$, the rate of roll $\dot{\varphi}$, the respective biases $b_{\dot{\psi}}^{(\mathrm{b})}, b_{\dot{\theta}}^{(\mathrm{b})}, b_{\dot{\varphi}}^{(\mathrm{b})}$ and noise terms $\varepsilon_{\dot{\psi}}, \varepsilon_{\dot{\theta}}$ and $\varepsilon_{\dot{\varphi}}$.

\section{EXTENDED KALMAN FILTER FOR TIGHT COUPLING}

In this section, we describe the joint estimation of the absolute receiver position and attitude using GNSS and INS measurements. Half cycle slips are assumed to be pre-corrected according to [3].

The set of measurements is divided into two types $\gamma \in$ $\{1,2\}$ of subsets: A first subset of GNSS measurements which is denoted by $s_{n}^{z}(\gamma=1)$ and a second subset of INS measurements described by $s_{n}^{z}(\gamma=2)$. The introduction of the subset type $\gamma$ enables an elegant notation as the state estimation based on GNSS and INS measurements can be described with the same expressions. At each epoch, there is only a subset of measurements (subset of satellites or gyroscope/ acceleration measurements) available. We write the set of available measurements at epoch $n$ as $z_{n}\left(s_{n}^{z}(\gamma)\right)$ with $s_{n}^{z}$ including the indices of the available measurements. The first subset is given by

$$
\begin{aligned}
z_{n}\left(s_{n}^{z}(1)\right)= & \left(\lambda \varphi_{1}^{\mathrm{T}}\left(t_{n}\right), \lambda \varphi_{2}^{\mathrm{T}}\left(t_{n}\right), \rho_{1}^{\mathrm{T}}\left(t_{n}\right), \rho_{2}^{\mathrm{T}}\left(t_{n}\right),\right. \\
& \left.f_{\mathrm{D}_{1}}^{\mathrm{T}}\left(t_{n}\right), f_{\mathrm{D}_{2}}^{\mathrm{T}}\left(t_{n}\right)\right)^{\mathrm{T}},
\end{aligned}
$$

where the SD carrier phases of the available satellites $k \in$ $\{1, \ldots, K\}$ are synchronized and corrected for cycle slips, i.e.

$$
\begin{aligned}
\lambda \varphi_{r}^{\mathrm{T}}= & \left(\lambda \varphi_{r}^{1, l}-c_{1, r}^{1, l}-\lambda / 2 \Delta \check{N}_{1, r}^{1, l}, \ldots\right. \\
& \left.\lambda \varphi_{r}^{K, l}-c_{1, r}^{K, l}-\lambda / 2 \Delta \check{N}_{1, r}^{K, l}\right)^{\mathrm{T}}
\end{aligned}
$$

The second subset includes the angular rate and acceleration measurements and is given by

$$
\begin{aligned}
z_{n}\left(s_{n}^{z}(2)\right)= & \left(\dot{\psi}^{(\mathrm{b})}\left(t_{n}\right), \dot{\theta}^{(\mathrm{b})}\left(t_{n}\right), \dot{\varphi}^{(\mathrm{b})}\left(t_{n}\right),\right. \\
& \left.a_{x}^{(\mathrm{b})}\left(t_{n}\right), a_{y}^{(\mathrm{b})}\left(t_{n}\right), a_{z}^{(\mathrm{b})}\left(t_{n}\right)\right)^{\mathrm{T}} .
\end{aligned}
$$

Similarly, we introduce two subsets of state parameters: The set of state parameters updated by GNSS measurements is written as

$$
\begin{aligned}
x_{n}\left(s_{n}^{x}(1)\right)= & \left(\vec{x}_{1}^{\mathrm{T}}\left(t_{n}\right), \vec{v}_{1}^{\mathrm{T}}\left(t_{n}\right), \psi\left(t_{n}\right), \dot{\psi}\left(t_{n}\right), \theta\left(t_{n}\right), \dot{\theta}\left(t_{n}\right),\right. \\
& \varphi\left(t_{n}\right), \dot{\varphi}\left(t_{n}\right), N_{1}^{\mathrm{T}}\left(t_{n}\right), N_{1,2}^{\mathrm{T}}\left(t_{n}\right), \\
& \Delta \rho_{\mathrm{MP}_{1}}^{\mathrm{T}}\left(t_{n}\right), \Delta \rho_{\mathrm{MP}_{2}}^{\mathrm{T}}\left(t_{n}\right), \\
& \left.b_{\dot{\psi}}^{(\mathrm{b})}\left(t_{n}\right), b_{\dot{\theta}}^{(\mathrm{b})}\left(t_{n}\right), b_{\dot{\varphi}}^{(\mathrm{b})}\left(t_{n}\right)\right)^{\mathrm{T}} .
\end{aligned}
$$

Note that the IMU biases of the angular rate measurements are also included in the state vector although not being directly observable by GPS. However, the Doppler measurements of the 2 nd receiver provide an unbiased information on the angular rates and, thereby, correct also the biases of the angular rates. These biases are directly observable by the gyroscope but can not be separated from the angular rates without GPS. The subset of state parameters updated by INS measurements is given by

$$
\begin{aligned}
x_{n}\left(s_{n}^{x}(2)\right)= & \left(\vec{x}_{1}^{\mathrm{T}}\left(t_{n}\right), \vec{v}_{1}^{\mathrm{T}}\left(t_{n}\right), \vec{a}_{1}^{\mathrm{T}}\left(t_{n}\right),\right. \\
& \psi\left(t_{n}\right), \dot{\psi}\left(t_{n}\right), \theta\left(t_{n}\right), \dot{\theta}\left(t_{n}\right), \varphi\left(t_{n}\right), \dot{\varphi}\left(t_{n}\right), \\
& b_{\dot{\psi}}^{(\mathrm{b})}\left(t_{n}\right), b_{\dot{\theta}}^{(\mathrm{b})}\left(t_{n}\right), b_{\dot{\varphi}}^{(\mathrm{b})}\left(t_{n}\right), \\
& \left.b_{a_{x}}^{(\mathrm{b})}\left(t_{n}\right), b_{a_{y}}^{(\mathrm{b})}\left(t_{n}\right), b_{a_{z}}^{(\mathrm{b})}\left(t_{n}\right)\right)^{\mathrm{T}} .
\end{aligned}
$$

The measurements of both observation types are used to update the state vector in a tight coupling. As the measurement rate of most low-cost gyroscopes and accelerometers is 100 $\mathrm{Hz}$ and, thus, higher than the measurement rate of most lowcost GNSS receivers, the state vector is updated more often by IMU than by GNSS measurements. The measurements are non-linear functions of the state vector due to the trigonometric relationship between the Euler angles and the baseline vector, i.e.

$$
z_{n}\left(s_{n}^{z}\right)=h_{n}\left(x_{n}\left(s_{n}^{x}\right)\right)+\eta_{z_{n}\left(s_{n}^{z}\right)},
$$

with $h_{n}$ being implicitly defined by the individual measurement models and $\Sigma_{z_{n}\left(s_{n}^{z}\right)}$ being the covariance matrix of the measurement noise $\eta_{z_{n}\left(s_{n}^{z}\right)} \sim \mathcal{N}\left(0, \Sigma_{z_{n}\left(s_{n}^{z}\right)}\right)$.

The dynamic behaviour of the vehicle and the temporal variations of the code multipath errors, gyroscope and acceleration biases is described by a linear state transition model:

$$
x_{n}\left(s_{n}^{x}\right)=\Phi x_{n-1}\left(s_{n-1}^{x}\right)+\eta_{x_{n}\left(s_{n}^{x}\right)},
$$

with the state transition matrix $\Phi$ and the covariance matrix $\Sigma_{x_{n}\left(s_{n}^{x}\right)}$ of the process noise $\eta_{x_{n}\left(s_{n}^{x}\right)} \sim \mathcal{N}\left(0, \Sigma_{x_{n}\left(s_{n}^{x}\right)}\right)$.

The state parameters are estimated with an extended Kalman Filter [1]. The tight coupling includes an update of the state vector with both inertial and GNSS measurements. The processing order is given by the measurement time stamp [4]. The Kalman filter includes the state prediction

$$
\hat{x}_{n}^{-}\left(s_{n}^{x}\right)=\Phi \hat{x}_{n-1}^{+}\left(s_{n-1}^{x}\right) .
$$

The covariance matrix of the predicted state is obtained from error propagation as

$$
\Sigma_{\hat{x}_{n}^{-}\left(s_{n}^{x}\right)}=\Phi \Sigma_{\hat{x}_{n-1}^{+}\left(s_{n-1}^{x}\right)} \Phi^{\mathrm{T}}+\Sigma_{x_{n}\left(s_{n}^{x}\right)},
$$


and the state update is given by

$$
\hat{x}_{n}^{+}\left(s_{n}^{x}\right)=\hat{x}_{n}^{-}\left(s_{n}^{x}\right)+K_{n}\left(z_{n}\left(s_{n}^{z}\right)-h_{n}\left(\hat{x}_{n}^{-}\left(s_{n}^{x}\right)\right)\right),
$$

with

$$
\Sigma_{\hat{x}_{n}^{+}\left(s_{n}^{x}\right)}=\left(1-K_{n} H_{n}\right) \Sigma_{\hat{x}_{n}^{-}\left(s_{n}^{x}\right)} .
$$

The convergence of the Kalman filter can be improved by using some a priori information on the state vector as described in [5]. We model this a priori information as

$$
\bar{x}_{n}=x_{n}+\eta_{\bar{x}_{n}} \quad \text { with } \quad \eta_{\bar{x}_{n}} \sim \mathcal{N}\left(0, \Sigma_{\bar{x}_{n}}\right),
$$

and extend the measurement vector to $\tilde{z}_{n}=\left(z_{n}^{\mathrm{T}}, \bar{x}_{n}^{\mathrm{T}}\right)^{\mathrm{T}}$.

The accuracy of the state estimate is further improved by fixing the DD ambiguities to integer numbers. We use the integer decorrelation of LAMBDA [6] and select the integer candidates based on the sum of squared errors [7]:

$$
\begin{aligned}
& \check{x}_{n}=\arg \min _{x_{n}}\left(\left\|z_{n}\left(s_{n}^{z}\right)-h_{n}\left(x_{n}\left(s_{n}^{x}\right)\right)\right\|_{\Sigma_{z_{n}\left(s_{n}^{z}\right)}^{-1}}^{2}+\right. \\
& \left.\left\|x_{n}\left(s_{n}^{x}\right)-\bar{x}_{n}\left(s_{n}^{x}\right)\right\|_{\Sigma_{\bar{x}_{n}\left(s_{n}^{x}\right)}^{-1}}^{2}\right) \text { s.t. } N_{1,2}^{k, l} \in \mathbb{Z} .
\end{aligned}
$$

The candidate with lowest error norm is chosen once the ratio of the MAP error norm between the second best and best candidate is sufficiently high.

\section{Measurement Results}

In this section, the proposed precise position and attitude determination is verified with real measurements from two low-cost GNSS receivers and an inertial sensor. First, the hardware set-up is described. Subsequently, the measurement and process noise assumptions are provided, and the obtained measurement results are analyzed. The measurement test was performed with a vehicle, on which the following hardware was mounted:

- 2 u-blox LEA 6T GPS receivers with $5 \mathrm{~Hz}$ data rate

- 2 L1 patch antennas mounted on the roof of a vehicle along its longitudinal axis with a baseline length of $l=1.2 \mathrm{~m}$ and $\sigma_{l}=1 \mathrm{~cm}$

- MPU 9150 inertial sensor from Invensense with a 3D gyroscope, a 3D accelerometer and a 3D magnetometer mounted on the roof of the vehicle providing measurements with a rate of $100 \mathrm{~Hz}$

- reference system: high-grade inertial sensor and geodetic GNSS receiver (tightly coupled)

\section{A. Measurement and process noise assumptions}

Tab. I and II include our measurement and process noise assumptions. The measurement statistics were determined in static conditions as follows: For the GNSS measurements, DDs were performed and analyzed over a (short) time period of 30 seconds. As static DDs can be well approximated by a linear model over such short time periods, a least-squares estimation of the coefficients of the linear model was performed. The standard deviations of the DD noises were then obtained from the RMS of the residuals of the least-squares estimation. The standard deviations of the undifferenced measurements were obtained by scaling of the standard deviations of the DDs. For the INS measurements, the same approach was used, i.e. measurements in static conditions were described by a linear model for a short period of time.

TABLE I

MEASUREMENT NOISE ASSUMPTIONS

\begin{tabular}{l|l} 
phase noise (undifferenced) & $\begin{array}{l}\sigma_{\varphi}=\{2 \mathrm{~mm} \ldots 4 \mathrm{~mm}\} \\
\text { depending on satellite elevation }\end{array}$ \\
code noise (undifferenced) & $\sigma_{\rho}=\{0.5 \mathrm{~m} \ldots 1 \mathrm{~m}\}$ \\
depending on satellite elevation \\
Doppler noise (undifferenced) & $\sigma_{f_{\mathrm{D}}}=\{1 \mathrm{~Hz} \ldots 10 \mathrm{~Hz}\}$ \\
depending on satellite elevation \\
angular rates & $\sigma_{\{\dot{\psi}, \dot{\theta}, \dot{\varphi}\}}=0.001 \mathrm{rad} / \mathrm{s}$ \\
acceleration & $\sigma_{\left\{a_{x}, a_{y}, a_{z}\right\}}=0.1 \mathrm{~m} / \mathrm{s}$
\end{tabular}

The process noise assumptions were chosen according to the dynamics of the vehicle, the temporal variation of multipath errors, and the sensor characteristics.

TABLE II

PROCESS NOISE ASSUMPTIONS

\begin{tabular}{l|l} 
acceleration & $\sigma_{a_{x}}^{(\mathrm{n})}=2.5 \mathrm{~m} / \mathrm{s}^{2}$ \\
& $\sigma_{a_{y}}^{(\mathrm{n})}=2.5 \mathrm{~m} / \mathrm{s}^{2}$ \\
& $\sigma_{a_{z}}^{(\mathrm{n})}=0.25 \mathrm{~m} / \mathrm{s}^{2}$ \\
derivatives of angular rates & $\sigma_{\ddot{\psi}}=25^{\circ} / \mathrm{s}^{2}, \sigma_{\ddot{\theta}}=25^{\circ} / \mathrm{s}^{2}$ \\
single difference ambiguities & $\sigma_{N}=0.25 \mathrm{cycles}$ \\
code multipath & $\{2 \mathrm{~m}, \ldots, 5 \mathrm{~m}\}$ \\
gyroscope biases & depending on satellite elevation \\
& $\sigma_{b_{\dot{\psi}}}=2 \cdot 10^{-7} \mathrm{rad} / \mathrm{s}$ \\
accelerometer biases & $\sigma_{b_{\dot{\theta}}}=2 \cdot 10^{-7} \mathrm{rad} / \mathrm{s}$ \\
& $\sigma_{b_{a_{i}}^{(\mathrm{b})}}=10^{-9} \mathrm{~m} / \mathrm{s}^{2}, i \in\{x, y, z\}$.
\end{tabular}

\section{B. Measurement results}

Fig. 1 shows the northern part of the vehicle's track as obtained from the absolute position estimates $\hat{\vec{x}}_{1}$.

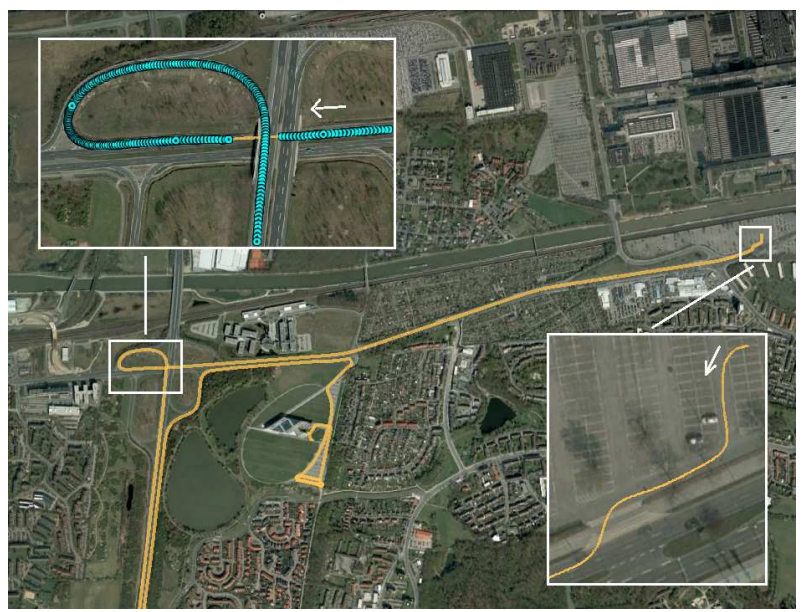

Fig. 1. Northern part of vehicle's track during test drive as determined by the proposed PPP with tight coupling of two low-cost GNSS receivers, a gyroscope and an accelerometer. The enlarged sections show the start and the passing below a bridge. Each blue point refers to a GPS-based state update.

The starting point is in the right part of the figure. The initial heading was $-98^{\circ}$, i.e. the car was oriented in western direc- 
tion. The track includes also a passing below a bridge. This passing is also enlarged with additional markers at every epoch with a GPS-based state update. The inertial measurements are used to update the state vector in between two GPS updates. One can observe a continuous path despite the GNSS signal interruption and increased multipath. As some satellites are at low elevation and as signals are also reflected, a GNSS-based state update can still be performed during the first meters of driving below the bridge. Once all tracking loops have lost lock, the re-acquisition also takes some time, which explains the distance between the end of the bridge and the first GPSbased state update after the bridge.

Fig. 2 shows the satellite-satellite single difference code multipath estimates for receiver 1 . Each curve represents one single difference. The multipath offsets of up to $15 \mathrm{~m}$ exceed the code noise level and are significantly correlated over time, which indicates the need of estimating these parameters.

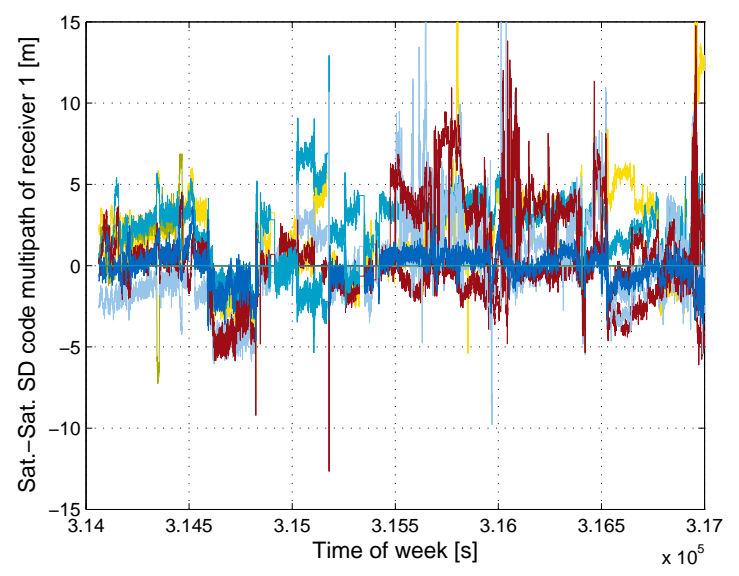

Fig. 2. Code multipath estimates for receiver 1: Each curve represents the code multipath for one satellite-satellite single difference measurement. One can observe a substantial correlation over time.

The estimated speed of the vehicle in Eastern direction is shown in Fig. 3. The difference between the speed estimate using low-cost GPS and INS hardware and the geodetic-grade GPS/ INS reference solution is in the order of only $0.1 \mathrm{~m} / \mathrm{s}$. It is also drift-free, which indicates a correct modeling and estimation of the acceleration biases.

Fig. 4 shows the heading of the vehicle. The heading estimate based on low-cost GPS and INS hardware closely follows the reference solution throughout the complete measurement period. The enlarged section refers to a bridge underpass. The heading estimate is continuous and the heading error remains less than $0.2^{\circ}$ despite the GNSS signal interruption. This indicates both a correct resolution of the DD integer ambiguities and a reliable correction of all cycle slips.

Fig. 5 shows the difference between the heading estimate obtained from the low-cost GPS/ INS hardware and the reference solution. The heading offset is less than $0.5^{\circ}$ for most of the time. As this error corresponds to a relative position error of only $1 \mathrm{~cm}$, the DD ambiguities were most likely resolved correctly.

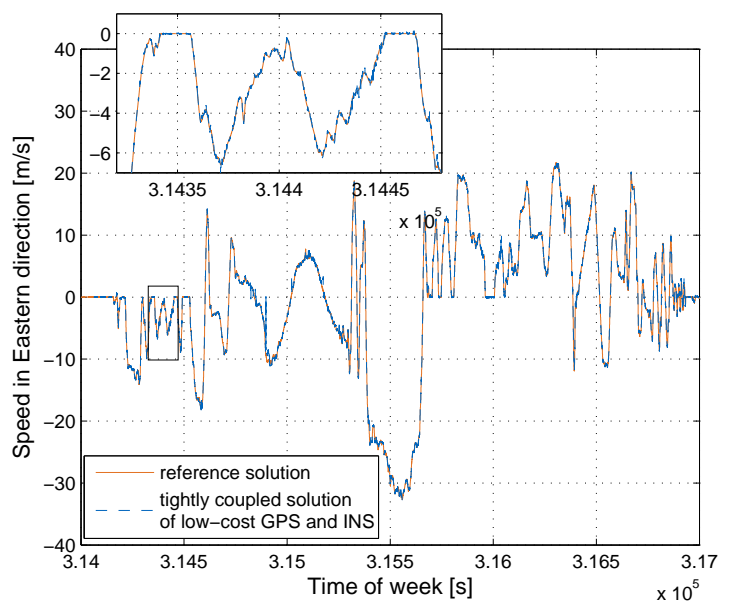

Fig. 3. The tightly coupled speed estimate of the low cost GPS/ INS is driftfree and differs by only $0.1 \mathrm{~m} / \mathrm{s}$ from the reference system, which indicates the correct modeling and estimation of acceleration biases.

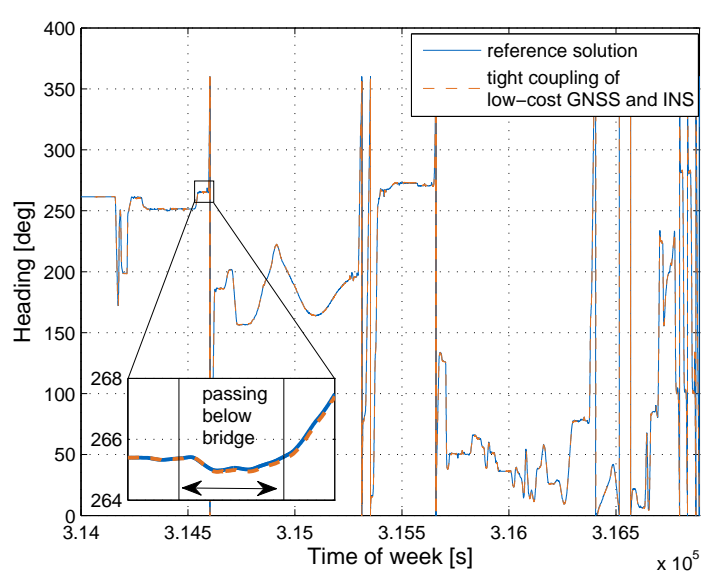

Fig. 4. The heading of the low-cost GPS/ INS hardware closely follows the curve of the reference solution in all environments.

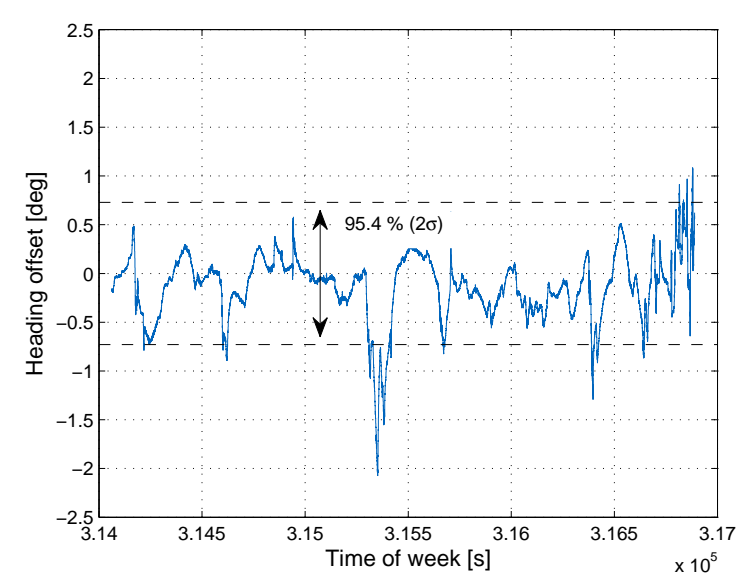

Fig. 5. The heading offset between the low-cost GPS/ INS hardware and the reference system is less than $0.5^{\circ}$ for most of the time, which indicates a correct resolution of the DD ambiguities. 
Fig. 6 shows the cumulative distribution of the heading error including all passages below bridges. The heading error is less than $0.27^{\circ}$ in $68.3 \%(1 \sigma)$ and less than $0.73^{\circ}$ in $95.4 \%(2 \sigma)$ of all epochs. The figure also shows the statistics of the heading estimate from a state of the art technique: As the carrier phase integer ambiguities are not resolved in current lowcost GNSS receivers, the heading is derived from the velocity, which is obtained solely from the Doppler measurements of a single GNSS receiver. A drawback of this approach is that no meaningful heading information can be obtained in static conditions. Moreover, the Doppler frequency can also not be tracked below bridges. Therefore, the availability of a precise heading is significantly lower than for the proposed tight coupling with carrier phase integer ambiguity resolution.

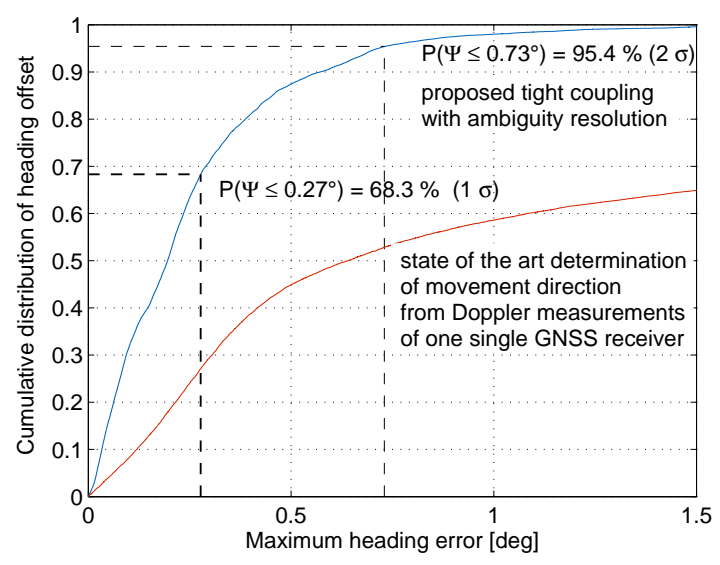

Fig. 6. Cumulative distribution of heading error: The heading error is less than $0.27^{\circ}$ in $68.3 \%(1 \sigma)$ and less than $0.73^{\circ}$ in $95.4 \%(2 \sigma)$ of all epochs.

Fig. 7 compares the rate of heading estimate using low-cost GPS/ INS hardware with the rate of heading of the reference system. The accuracy is in the order of $0.1^{\circ} / \mathrm{s}$. A similar performance was observed for the rate of pitch.

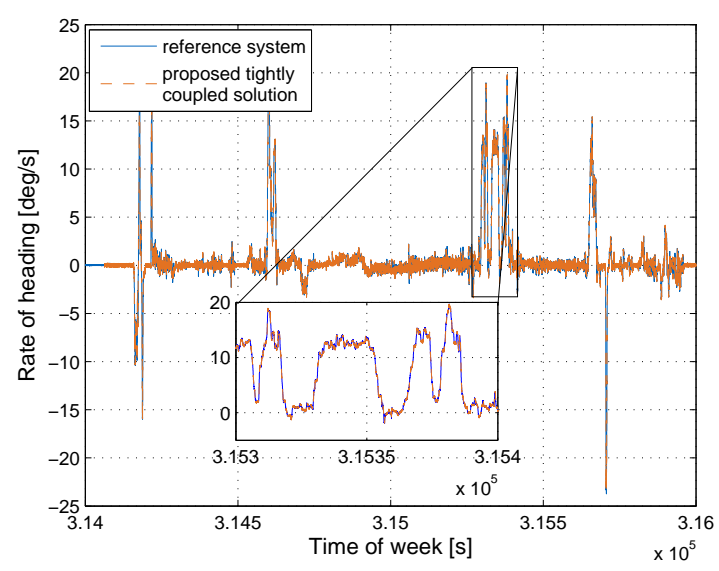

Fig. 7. Comparison of rate of heading estimate of low-cost GPS/ INS system with reference system. The accuracy is in the order of $0.1^{\circ} / \mathrm{s}$.

Fig. 8 shows the gyroscope bias for the rate of heading measurement. The bias varies by up to $\left(0.01^{\circ} / s\right) / 60 \mathrm{~s} \approx 2$. $10^{-6} \mathrm{rad} / \mathrm{s}^{2}$ during the measurement period. For the bias of the rate of pitch measurement, we observed a similar order of magnitude.

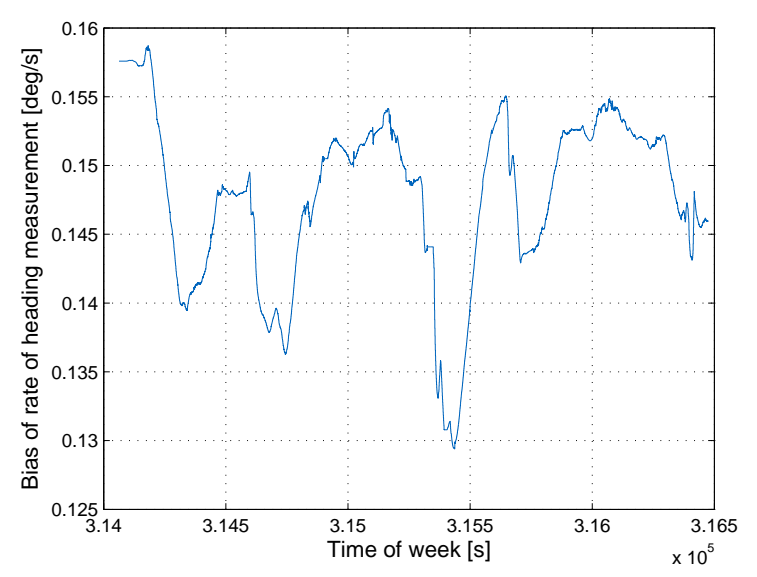

Fig. 8. Bias of rate of heading measurement. The gyroscope bias varies by up to $\left(0.01^{\circ} / \mathrm{s}\right) / 60 \mathrm{~s} \approx 2 \cdot 10^{-6} \mathrm{rad} / \mathrm{s}^{2}$.

\section{CONCLUSION}

In this paper, a tightly coupled position and attitude determination method was developed for low-cost GNSS receivers, an accelerometer and a gyroscope. The method includes a synchronization correction to improve the positioning accuracy and to restore the integer property of double difference GNSS carrier phase ambiguities.

The sensor fusion was performed with an extended Kalman filter, which estimates the position, velocity, acceleration, attitude, angular rates, single and double difference integer ambiguities, single difference code multipath delays, and biases of the angular rate/ acceleration measurements. Cycle slips are reliably corrected by predicting the position with the inertial measurements. The proposed method was verified in a test drive with two u-blox LEA 6T GPS receivers. We achieved a heading accuracy of $\sigma_{\psi}=0.25^{\circ} /$ baseline length [m].

\section{REFERENCES}

[1] R. Brown and P. Hwang, Introduction to Random Signals and Applied Kalman filtering, John Wiley, 4th edition, 2012.

[2] P. Henkel, P. Berthold and J. Kiam, Calibration of Magnetic Field Sensors with two mass-market GNSS receivers, Proc. of 11-th IEEE Workshop on Positioning, Navigation and Communication (WPNC), Dresden, Germany, 2014.

[3] P. Henkel and N. Oku, Cycle slip detection and correction for heading determination with low-cost GPS/ INS receivers, Proc. of VIII Hotine Marussi Symposium, IAG, Rome, Italy, Jun. 2013.

[4] P. Groves, Principles of GNSS, Inertial, and Multisensor Integrated Navigation Systems, 2nd edition, Artech House, 2013.

[5] P. Henkel, P. Jurkowski and C. Günther, Differential Integer Ambiguity Resolution with Gaussian a priori knowledge and Kalman filtering, Proc. of 24th ION GNSS, Portland, USA, pp. 3881-3888, Sep. 2011.

[6] P. Teunissen, The least-squares ambiguity decorrelation adjustment: a method for fast GPS ambiguity estimation, J. of Geodesy, vol. 70, pp. 65-82, 1995.

[7] P. Henkel and J. Kiam, Maximum A Posteriori Probability Estimation of Integer Ambiguities and Baseline, Proc. of 55-th IEEE Symp. ELMAR, pp. 353-356, Zadar, Croatia, Sep. 2013. 